\title{
Tangence
}

\section{Les soeurs ennemies : Gabrielle et Adèle Roy*}

\section{Lori Saint-Martin}

Numéro 62, avril 2000

Parentèle

URI : https://id.erudit.org/iderudit/008173ar

DOI : https://doi.org/10.7202/008173ar

Aller au sommaire du numéro

Éditeur(s)

Presses de l'Université du Québec

ISSN

0226-9554 (imprimé)

1710-0305 (numérique)

Découvrir la revue

Citer cet article

Saint-Martin, L. (2000). Les soeurs ennemies : Gabrielle et Adèle Roy*.

Tangence, (62), 50-72. https://doi.org/10.7202/008173ar d'utilisation que vous pouvez consulter en ligne.

https://apropos.erudit.org/fr/usagers/politique-dutilisation/ 


\section{Les sœurs ennemies: Gabrielle et Adèle Roy* Lori Saint-Martin, Université du Québec à Montréal}

Lorsqu'il fit constuire la nôtre, mon père prit comme modèle la seule autre maison qui se trouvait alors dans cette petite rue Deschambault sans trottoir encore, frâiche comme un sentier entre des buissons d'aubépine, et, en avril, toute remplie du chant des grenouilles. [...] Il eut l'air heureux en arrivant devant notre maison; d'un coup d'œil il embrassa les trois petits pommiers en fleurs, la galerie spacieuse avec sa rangée de chaises berceuses, la couleur frâiche de la peinture et jusqu'à ma frimousse qui le guettait.

Gabrielle Roy, Rue Deschambault, Boréal, 1993, p. 9, p. 15

Au cours du printemps 1905, le père fit construire sa maison dans la rue Deschambault. Nous y emménagêames le 22 août.

La mère acheta peu de meubles, trois lits, une commde, un fourneau et une berceuse. Cependant, elle fit mettre un tapis de haute laine dans le salon, et un autre étroit, sur les marches de l'escalier, des linoléums sur le plancher du cabinet de travail du père, de la salle à manger, de la cuisine et des corridors. Le père fit l'emplette d'un sofa recouvert de cuir, d'un pupitre à cylindre et d'un fauteuil en bois [...] Toutes ces choses embellirent la maison du père et lui donnèrent un visage plus gai, plus agréable et plus intime.

Marie-Anna A. [Adèle] Roy, Le miroir du passé, Québec/Amérique, 1979, p. 20

* Le présent article s'inscrit dans le cadre d'une subvention du FCAR et de l'Université du Québec à Montréal, intitulée "Pour une nouvelle Gabrielle Roy". Je tiens à remercier M. François Ricard des informations qu'il m'a fournies à propos des deux sœurs Roy et de ses judicieuses remarques à propos de mon article. 
Sauf peut-être chez les amateurs d'histoire du Manitoba, à qui elle fournit de précieux renseignements sur la colonisation de cette province, l'œuvre d'Adèle Roy n'a suscité à peu près aucun intérêt. Si l'on a publié certains de ses livres, dont ses deux romans, Le pain de chez nous (1954) et Valcourt ou la dernière étape (1958), ainsi que Le miroir du passé, son autobiographie (1979), c'est en raison de sa parenté avec Gabrielle Roy, dont elle est la sœur aînée et la marraine ${ }^{1}$. Toute sa très longue vie (elle n'est morte qu'en 1998, à 105 ans), Adèle aura donc vécu à l'ombre de sa sœur, plus jeune, plus belle, plus célèbre, plus riche, et, imagine-t-elle, plus heureuse qu'elle, plus aimée de surcroît de leur mère, qu'Adèle a pourtant adorée et dont elle estime s'être mieux occupée. Lorsqu'on souffre d'hypersensibilité et prend la mouche devant toute offense réelle ou perçue, il y a là matière à nourrir une violente et éternelle haine. En même temps que cette haine, naissent chez Adèle une obsession passionnée pour Gabrielle que même la mort de celle-ci, survenue en 1983, ne pourra apaiser, et un désir d'appeler sur elle le regard de cette sœur trop choyée par la vie, un désir d'être enfin vue d'elle et reconnue, voire redoutée et haïe, à défaut d'être aimée. Naît surtout, parallèlement à une tenace volonté d'autojustification, le besoin de se démarquer de cette sour qui représente pourtant tout ce qu'elle aurait voulu être. Elle y parviendra en édifiant, dans l'ambivalence et le paradoxe, une vision de l'écriture qui donnera tort à Gabrielle tout en faisant d'elle-même l'écrivaine véritable. Ainsi, à défaut d'avoir connu la célébrité et la richesse, Adèle aura du moins la satisfaction d'avoir eu, du début à la fin, raison. "Bonne fille" de la mère, elle écrira aussi les "bons livres", alors que Gabrielle, mauvaise fille, sera aussi mauvaise écrivaine.

$\mathrm{Au}$ cour de la querelle qui a séparé les deux femmes (qui ont rarement été proches, sinon pendant une brève idylle suivant le retour d'Europe de Gabrielle, durant ses années de journalisme), on retrouve la guerre du bien et du mal, du réel et de la fiction, du don et de l'égoïsme. "Faire de la fiction" deviendra un terme péjoratif dans la bouche d'Adèle, fait ironique pour une femme de lettres. L'étude des relations orageuses entre les sœurs

1 Axé sur la célébrité de Gabrielle, le texte de l'endos du Miroir du passé annonce une description "sans complaisance" qui révélera "l'envers du décor" de la maison de la rue Deschambault; un passage concernant Gabrielle y est reproduit. Autrement dit, c'est le nom de Gabrielle plutôt que celui d'Adèle qui sert d'appât. 
52

se fera ici en trois temps: après avoir examiné la perspective d'Adèle, puis celle de Gabrielle, nous verrons ce qui rapproche les deux sœurs dans leur division même, ce qui les réunit, malgré elles, par-delà la haine et l'incompréhension.

\section{ADÈLE}

Afin de mieux comprendre le litige entre les deux sœurs, disons quelques mots de la vie d'Adèle, bien moins connue que celle de Gabrielle ${ }^{2}$. Née en 1893, cinquième enfant de la famille Roy ${ }^{3}$, Adèle devra, très tôt, seconder sa mère dans toutes ses tâches domestiques; elle fait donc l'apprentissage précoce du travail et des soucis. À l'instar de sa sœur aînée, Anna, et comme le fera Gabrielle plus tard, Adèle décide assez jeune de rompre avec le modèle maternel : énergique, déterminée et forte, elle désire apprendre, voyager, faire sa marque. Elle voudrait obtenir son baccalauréat ès arts, mais doit y renoncer devant divers obstacles, dont l'opposition réunie du clergé et de sa famille (après vingt ans de cours du soir ou par correspondance, elle l'obtiendra enfin en 1934). Devenue enseignante, elle obtient sa première école en 1914 et découvre les plaisirs défendus : la danse, l'alcool, la dissipation. Elle découvre aussi l'amour, de courte durée toutefois: mariée en 1914, elle se sépare de son mari après quelques semaines à peine, provoquant un scandale dont la honte la suivra longtemps et aigrira son caractère. Pendant des années, elle continuera d'enseigner, travaillant dans des villages perdus, loin de la vie artistique et intellectuelle dont elle rêve; elle se déplace presque chaque année, à la recherche d'une école meilleure ou parce qu'elle a été renvoyée. Elle sera donc toujours étrangère, toujours nomade, et perpétuellement insatisfaite. Deux tentatives de s'établir à Montréal échouent. Elle achète une terre à Tangent, la cultive quelques années, la vend et regrette aussitôt de s'en être défaite. Ce n'est qu'à la retraite, après 1959, qu'elle réalisera enfin un rêve de jeune fille: voir l'Europe. À partir des années 1950, elle donne aussi libre cours à un autre désir longtemps freiné: celui d'écrire. Un livre de sou-

2 Voir, pour ce qui suit, Paul Genuist (avec la collaboration de Monique Genuist), Marie-Anna Roy, une voix solitaire, Saint-Boniface, Des plaines, 1992, et François Ricard, Gabrielle Roy, une vie, Montréal, Boréal, 1996.

3 Joseph et Anna la précèdent, ainsi qu'un garçon mort à 3 mois et Agnès, que la méningite a emportée en 1906, à l'âge de 14 ans. 
venirs d'enfance romancés, Le pain de chez nous (1954), est suivi de Valcourt ou la dernière étape (1958), puis de divers ouvrages portant sur l'histoire de la colonisation manitobaine, où elle laisse s'épancher son sentiment patriotique et son amour des traditions. Adèle publie pour l'essentiel à compte d'auteur ou encore grâce à la contribution financière d'organismes québécois et manitobains francophones et de bienfaiteurs privés, dont Gabrielle Roy ${ }^{4}$. Publiés ou non - Adèle remet à certaines institutions et à quelques particuliers des manuscrits qui du reste se recoupent souvent entre eux —, les écrits d'Adèle ressassent encore et toujours ses obsessions: sa bonté et l'égoïsme de Gabrielle, le culte des ancêtres, le souvenir sacré de la rue Deschambault. Malgré son dévouement et son travail acharné, jamais la gloire et la fortune rêvées ne se matérialiseront. Les sentiments dominants, chez Adèle, seront donc la désillusion et l'envie, bien sûr, et, surtout, un profond sentiment d'injustice. D'où le besoin persistant de se justifier et de condamner les autres: les reproches visent à divers degrés tous les frères et sœurs d'Adèle, et, en tout premier lieu, Gabrielle.

\section{Adèle justicière : l'argent, la dette et la « bonne fille »}

L'un des plus puissants moteurs de l'écriture d'Adèle (trait d'ailleurs qui la rapproche de sa sour, nous y reviendrons) est précisément ce désir d'autojustification et d'autoencensement. Il faut dire la vérité, rétablir les faits, affirmera-t-elle, contredire l'image souriante que Gabrielle a donnée d'elle-même dans ses livres; récupérer elle-même le beau rôle, pourrait-on dire plutôt, montrer qu'elle a toujours été la meilleure, la plus généreuse, le seul enfant Roy doté d'une quelconque noblesse de cœur.

La lecture du Miroir du passé (1979), l'autobiographie d'Adèle, suscite une impression de profonde tristesse. Tant de déceptions, tant de rancune s'y étalent qu'on a pitié de celle qui écrit, sans qu'on se laisse toucher par son écriture terne et sans finesse, remplie de clichés et de maladresses. Aucun de ses frères et sœurs ne trouve grâce aux yeux d'Adèle. Les frères, volages, dépensiers, parfois fourbes, suscitent une kyrielle d'adjectifs péjoratifs (Germain, par exemple, est "capricieux, exigeant, égoïste, malendurant et

4 Paul Genuist, op. cit., p. 52. 
grippe-sou ") mais peu d'intérêt véritable. Les sœurs, en revanche, occupent dans l'imaginaire d'Adèle une place prépondérante. Clémence est une pauvre fille bornée et entêtée, Bernadette, trop nerveuse et trop faible, a fui les responsabilités familiales en devenant religieuse (Adèle se réconciliera avec Bernadette vers la fin de la vie de celle-ci et regrettera les jugements impitoyables qu'elle a portés sur sa cadette), Anna est mesquine, envieuse, égocentrique, obsédée par l'argent, maladivement susceptible et incapable de s'entendre avec les autres - c'est d'ailleurs son propre portrait qu'Adèle esquisse là sans s'en rendre compte. Et, surtout, Gabrielle. Depuis toujours, "Cad" (pour "Cadette") est gâtée, capricieuse, égoïste et manipulatrice, bien qu'elle se croie bonne et généreuse. Jeune fille déjà, elle se fait donner de l'argent par Adèle pour offrir des cadeaux à la famille, mais le garde pour elle; survient alors la première rupture entre les deux soeurs. Dès qu'elle le pourra, on verra Gabrielle "abandonn[er] sa pauvre mère infirme, attirée par le mirage d'une brillante carrière d'artiste qui ferait pleuvoir dans ses mains avides des centaines de milliers de dollars!» (MP, p. 123). Après la mort de la mère, elle refusera obstinément d'aider ses søurs dans le besoin, sinon en leur remettant parfois, comme à contre-cour, quelques dollars, elle qui, avant de partir pour l'Europe, affirmait vouloir devenir riche à seule fin de sortir les siens de la misère.

Quant à Adèle elle-même, Le miroir du passé fait surtout le récit de ses innombrables bontés. Elle seule a donné des soins et de l'argent aux parents; tous les autres en ont réclamé, en cajolant les vieux comme Gabrielle, en les intimidant comme Anna ou en les volant comme Germain. L'argent est évoqué à presque toutes les pages du livre: Adèle se souvient du prix du calorifère acheté par le père en 1916 (750,00 \$); elle signale qu'à la mort de son père, elle a donné 490,00 \$ à la mère, que, peu après, elle lui a "gliss[é] 22 \$ dans la main" (MP, 114), et ainsi de suite. Le simple fait de retenir des chiffres aussi précis $(490,00 \$$, pas 500,00 \$), que sa mémoire les ait réellement conservés ou qu'elle les ait notés et gardés pendant un demi-siècle, montre, au-delà d'une pauvreté bien réelle et d'une générosité non moins authentique, une volonté d'autojustification et une capacité de rancune peu ordinaires. Parfois, à force de vouloir paraître magnanime

5 Marie-Anna A. [Adèle] Roy, Le miroir du passé, Montréal, Éditions Québec/Amérique, 1979, p. 66. Cette œuvre sera désignée dans le corps du texte par l'abréviation suivante: $M P$. 
sans rien omettre des injustices qu'elle a subies, Adèle tombe dans des contradictions étranges: elle dit, par exemple, avoir brûlé, en 1923, une lettre particulièrement venimeuse d'Anna, mais, près de 60 ans plus tard, elle en cite dix lignes entre guillemets, comme si elle la possédait encore. En fait, si Adèle chérit tant ses souvenirs et les étale sans cesse, c'est parce qu'elle a été — ou qu'elle s'est créée —, sans reproches ou presque: généreuse avec l'argent, prodigue de sages conseils, elle a été le principal soutien de sa mère et l'unique confidente et consolatrice de son père. Elle ne se fait que deux reproches: avoir un jour fait dormir sa mère sur un mauvais lit ( $M P$, p. 91), tort qu'elle a réparé dès la visite suivante en lui sacrifiant sa propre chambre, et avoir refusé une fois de prêter de l'argent à Germain à la demande du père (p. 103). Mais, pour l'essentiel, Adèle se recrée seule admirable, seule généreuse, seule "bonne fille" envers leurs parents. Les autres, à des degrés divers, seront les "mauvaises filles ». Et surtout Gabrielle, bien entendu, celle qui a eu la cruauté et l'audace de partir, celle qui a réussi ${ }^{6}$.

Où sont les torts réels dans cette triste histoire? Peut-on le savoir, importe-t-il même de le savoir? Fait intéressant, dans leurs autobiographies respectives, les sœurs ennemies brossent essentiellement le même portrait d'Anna, de Clémence et des frères. Seul leur portrait l'une de l'autre varie du tout au tout, chacune dépeignant l'autre sous un mauvais jour et cherchant à se donner le beau rôle. Adèle a sûrement donné plus d'argent à sa mère, proportionnellement à ses revenus de l'époque, aussi maigres que ceux de Gabrielle ${ }^{7}$. En revanche, comme Gabrielle le fait dire à sa mère dans La détresse et l'enchantement ${ }^{8}$, tous les autres

6 Ce portrait de famille trouve sa première expression dans le roman autobiographique d'Adèle, Le pain de chez nous, où, sous les traits de Domitilde, elle se dépeint comme celle qui a continuellement secouru sa mère, alors que Gaétane (Gabrielle) l'a abandonnée sans remords. Durant le quart de siècle qui sépare le roman de l'autobiographie, la bonne conscience d'Adèle n'a fait que s'affermir.

7 François Ricard, op. cit., fait remarquer que même lorsqu'elle se mettra à toucher, du Bulletin des agriculteurs, un salaire de 250,00 \$ par mois, Gabrielle n'augmentera pas la mensualité de 10,00 \$ qu'elle envoie à sa mère.

8 C'est probablement la vérité puisque dans Le pain de chez nous (Montréal, Éditions du Lévrier, 1954, p. 233), Adèle fait dire à la mère: "Que veux-tu? À vingt-sept ans elle est libre d'agir à sa guise! Que serais-je devenue si elle s'était mariée à vingt ans comme Léona?". Cette œuvre sera désignée dans le corps du texte par l'abréviation suivante: $P C N$. 
enfants, y compris Adèle, ont quitté très tôt le foyer familial, que ce soit pour se marier, pour enseigner au loin ou simplement, comme quelques-uns des garçons, pour vagabonder; Gabrielle seule est restée aux côtés de la mère jusqu'à l'âge de 28 ans. Adèle elle-même raconte que lorsqu'elle revient au foyer après de longues absences, il arrive que sa mère ne la reconnaisse pas et lui adresse la parole en anglais ( $P C N$, p. 210). Qu'est-ce qui comble davantage une mère: l'argent ou la présence quotidienne? Adèle a donné le premier, Gabrielle la seconde. Mais, curieusement, bien qu'Adèle et tous les autres soient donc partis plus tôt que Gabrielle, celle-ci, seule, sera accusée, jugée et condamnée. La vie familiale est abondamment tissée de tels jugements sans réplique, souvent, comme ici, en partie injustes, ainsi que de telles fictions arbitraires qui deviennent vraies du moment que toute la famille les croit telles. Encore là, on se retrouve devant une construction d'Adèle, le vieux jeu "bonne fille-mauvaise fille".

Il est vrai que, pour se lancer comme l'a fait Gabrielle à la poursuite d'un avenir encore sans forme, pour renoncer à la sécurité et sauter dans le vide, il faut une force de caractère peu commune. Gabrielle elle-même se trouvera courageuse, autant que téméraire, et affirmera plus tard que sa famille a cherché par tous les moyens à bloquer sa route ${ }^{9}$; Adèle susurre : égoïsme, ambition, folie des grandeurs. Adèle dira du reste avoir souhaité, elle aussi, réaliser ses propres projets; seulement, par amour pour sa mère, elle en aura remis à plus tard l'exécution, afin de donner tout son argent à Mélina (PCN, p. 125). Que ce soit par bonté de cour ou encore par inertie et crainte d'échouer, Adèle n'a pas tout risqué, comme l'a fait Gabrielle en partant en Europe, en quittant son emploi sûr et en s'établissant à Montréal sans savoir d'abord comment elle y gagnerait sa vie. Et elle le regrette, tout en s'en félicitant. Elle aurait bien voulu être celle qui part sans un regard pour ce qu'elle laisse derrière. Si, comme Adèle l'écrira sentencieusement à Gabrielle, "Le plus grand bonheur de la vie, et, à vrai dire, le seul, est de faire du bien à ses semblables" (MP, p. 119), pourquoi a-telle été si malheureuse? Il semble donc évident que cette construction de la "bonne fille" et de la "mauvaise fille" sert surtout à con-

9 Une lettre dans laquelle Adèle l'informe, "sans malice", qu'elle "[n'a] pas les talents requis pour devenir une vedette" $(M P, 119)$, tend à donner raison à Gabrielle. Sous la mise en garde amicale, on sent bien le désir de freiner une ambition menaçante pour l'entourage. 
soler Adèle d'avoir manqué de force et d'audace. Car il est relativement facile de se dire, une fois la vieillesse venue: j'aurais pu être un grand écrivain si je ne m'étais pas tant sacrifiée pour aider ma famille. On s'épargne ainsi les années d'errance, de misère et de doute qu'a connues Gabrielle, ainsi que le travail acharné qu'il faut pour devenir un écrivain authentique. Adèle a donc pu voir la vie de Gabrielle comme l'envers de la sienne, tout ce qui n'a pas porté fruit dans son cas ayant réussi à merveille à Gabrielle. Là où Adèle a dû seconder sa mère dès sa jeunesse, Gabrielle, la benjamine, choyée de tous, n'a pas eu à lever le petit doigt; très tôt, elle obtient un poste convoité à Saint-Boniface, si bien qu'elle n'aura pas à courir toute sa vie les villages perdus. Adèle détestait l'enseignement, "cette vocation sans gloire et sans mérite" ( $M P$, p. 74), mais a dû y demeurer 35 ans; elle n'a pu visiter l'Europe qu'à la retraite. Alors que Gabrielle, elle, s'est libérée du monde étriqué qui était le leur, a vécu en France et en Angleterre avant la trentaine et, non contente de vivoter grâce au journalisme, est devenue riche et adulée. Qui plus est, ce succès lui vient en partie de la description (fort romancée) qu'elle fait de l'enseignement et d'elle-même enseignante, dans La Petite Poule d'Eau d'abord, dans Ces enfants de ma vie ensuite; ce monde qu'elle peint sous un jour idéalisé, cet univers où les élèves sont doux et prévenants plutôt que rebelles ou paresseux comme ceux qu'a connus Adèle, elle s'est empressée toute jeune de le quitter pour toujours. Or les romans autobiographiques de Marie-Anna Adèle ne connaissent aucun succès, bien qu'ils évoquent le même monde. Pourtant, Adèle n'a-t-elle pas travaillé aussi fort que Gabrielle, sans jouir des mêmes avantages? Elle a passé ses nuits à lire les grands auteurs, à étudier les langues anciennes, et à écrire. Est-ce de sa faute si elle n'a pas eu, comme Gabrielle, un "bon ami" (Henri Girard) qui l'a lancée dans les milieux journalistiques et radiophoniques et qui a pratiquement écrit ses livres (MP, p. 141)?

Ainsi se construit le monstre Gabrielle: égoïste, sans cour, sans grandeur d'âme malgré les hautes valeurs humanitaires qu'elle loue dans ses livres, non sans talent mais surestimée de tous et surtout imbue d'elle-même, célèbre "grâce au mythe forgé aux trois quarts par la presse à grand tirage" ( $M P$, p. 171). Ayant donc fait de Gabrielle la "mauvaise fille", Adèle entreprendra de définir une esthétique littéraire qui en fera aussi une "mauvaise écrivaine", tandis qu'Adèle, elle, sera celle qui écrit les bons livres pour les bonnes raisons. 
58

\section{Vérité et mensonge : les bonnes et les mauvaises œuvres}

Filles d'une mère conteuse (bien que les récits oraux du père soient également évoqués, l'écriture, chez les Roy, se transmet essentiellement par la voie matrilinéaire), Adèle et Anna auraient voulu, elles aussi, écrire. Adèle n'a-t-elle pas suivi pendant quelques années des cours par correspondance de "l'École A.B.C. de Rédaction de Paris ${ }^{10}$ "? Gabrielle signale que ses sours, plus âgées, auraient eu tout le temps de faire leur marque avant elle; qu'elles n'aient rien fait montre qu'elles n'étaient pas prêtes, comme Gabrielle, à se donner à la littérature comme à une ascèse. Anna et Adèle affirment plutôt que Gabrielle a raillé leurs efforts et qu'elle a refusé de leur trouver des appuis dans les milieux littéraires où elle faisait la pluie et le beau temps; Adèle raconte même que Gabrielle, non contente de massacrer les épreuves de son premier roman, Le pain de chez nous, "saccageant mon jardin littéraire, piétinant mes plus brillantes fleurs, sans aucun ménagement" (MP, p. 204), l'a suppliée de n'en rien publier, puis s'en est inspirée pour faire paraître, dès l'année suivante, Rue Deschambault (dont, comble d'injustice, la traduction lui vaudra le prix du Gouverneur général).

Les reproches faits à Gabrielle visent donc autant ses ouvres que ses actions. À cet égard, un texte d'Adèle en dit long sur sa construction de Gabrielle en "mauvaise écrivaine". Dans Les Capucins de Toutes-Aides (1977), ouvre qui porte presque le même titre que la troisième partie de La Petite Poule d'Eau, Adèle interrompt son récit des hauts faits de missionnaires belges au Manitoba pour s'attaquer aux faussetés que contient justement le deuxième roman de sa sœur ${ }^{11}$. Elle commence, cinglante, par rétablir les faits :

Dépouillée de toute fiction et de poésie, l'histoire se résume simplement. Vers la fin du $\mathrm{XIX}^{\mathrm{e}}$ siècle et au début du $\mathrm{Xx}^{\mathrm{e}}$ siècle, des colons français s'établirent à Sainte-Rose du Lac et plus au nord sur les bords du lac Manitoba. Deux soi-disant "nobles" louèrent une île à 65 milles au nord de Sainte-Rose pour y faire

10 Paul Genuist, op. cit., p. 43.

11 Son entrée en matière, brusque pour dire le moins (le chapitre débute ainsi : "Le second roman de Cad, "La Petite Poule d'Eau", se composait de 3 nouvelles ou contes..." [p. 66]), trahit involontairement une obsession pour Gabrielle et pour ses écrits qui dément le patient travail de démolition littéraire auquel Adèle se livre dans tout le chapitre. 
l'élevage des animaux [...] Tout marcha bien pendant quelques années; mais un hiver la maladie s'abattit sur les bœufs et en fit mourir un bon nombre, causant de lourdes pertes aux propriétaires. Ceux-ci abandonnèrent leur ranch et quittèrent le pays. Après leur départ, M. Joseph Jeannotte acheta de la Banque à Winnipegosis le morceau de terrain, au centre de l'île, où s'élevaient le corps de logis et les dépendances [...] Il engagea son oncle, Albert Côté, pour gérer son ranch — de 1930 à 1938. La famille Côté en 1930 comprenait, outre le père, la mère et sept enfants [...] Les parents écrivirent au ministre de l'éducation, lui demandant une institutrice. Une école particulière fut aménagée dans l'ancienne maison "des maîtres" et M. William E. Mason arriva le premier mars 1935 . Il enseigna à raison de $\$ 35.00$ par mois, logé et nourri, jusqu'au début de septembre. L'école n'avait aucun nom, parce que la véritable école de la région, "The Water Hen School, Number 1955", se trouvait à une vingtaine de milles à l'est, au centre du hameau "Waterhen". Le maître d'école [...] n'étant pas amateur de chasse et de pêche, ne perdait pas son temps à fourbir ses armes [...] Il travaillait avec zèle et dévouement à l'instruction de ses élèves, désireux d'acquérir de l'expérience et d'obtenir une bonne note de l'inspecteur d'école ${ }^{12}$.

Voilà donc pulvérisées, croit Adèle, les fabrications de Gabrielle, peu soucieuse de la vérité (pour qui connaît La Petite Poule d'eau, cette rectification vise chacune de ses scènes clés, de la recherche d'un nom pour l'école à l'arrivée magique de $\mathrm{M}^{\text {lle }}$ Côté dans l'île). Dans le même texte, Adèle reproche à Gabrielle de réunir des éléments disparates - la petite fille "surprise" est née non pas à Mme Côté mais à une cousine des Roy, Luzina faisant de la couture pour les prêtres rappelle Mélina Roy —, de situer dans tel décor une scène qui s'est déroulée ailleurs, de modifier la chronologie, de tracer du propriétaire de l'île, pourtant fort sympathique en réalité, un portrait accablant, de déshonorer l'image des prêtres en transformant le capucin en marchand de fourrures. De plus, faute d'avoir fait une "étude sérieuse et profonde de la topographie des lieux" (CTA, p. 69), Gabrielle commet des erreurs grotesques, car elle aurait dû savoir "que les poules de prairies "gelinottes" n'émigrent pas au sud à l'automne; que la toundra touche au cercle polaire [...] et que pour atteindre

12 Les Capucins de Toutes-Aides, Éditions franciscaines, Montréal, 1977, p. 66, 68. Cette ouvre sera désignée dans le corps du texte par l'abréviation suivante: CTA. 
60

l'île à Bessette en venant de Meadow Portage, il fallait traverser "la Petite Poule d'Eau" (Little Waterhen) et non "The West Waterhen” "(CTA, p. 69). Faute donc de profondeur et de sérieux, triomphera une imagination qui "donne dans l'exagération" (CTA, p. 71): "tout le reste est pure invention et baigne dans le fantaisiste" (CTA, p. 63) et la "déformation d'un caractère [celle du capucin], que rien ne laissait prévoir, ni pressentir, est illogique, invraisemblable et baigne dans le fantaisiste et l'onirisme!" (CTA, p. 74). Et pourtant - suprême injure pour les amoureux de la vérité —, Radio-Canada "a fait en 1970 une propagande absurde et outrée au sujet du roman" (CTA, p. 74).

À la fin de cette tirade, nous lisons ceci :

MAINTENANT

TIRONS LE

RIDEAU

SUR LA

FICTION

LEVONS-

LE SUR

LA

RÉALITÉ (75).

Reprend alors, sans transition, le récit d'Adèle. Autrement dit: Gabrielle est morte, vive Adèle. La Vérité a triomphé du mensonge: Gabrielle invente et triche; moi, Adèle, je dis la vérité.

Sous cette apparence de pédanterie, sous ce ton persifleur, se cache bien sûr de l'envie, mais justifiée d'une manière originale: Gabrielle ne mérite pas le succès qu'elle a eu, car non seulement c'est une mauvaise personne, mais elle ne dit pas la vérité dans ses livres. Cette lecture, en apparence naïve, prête à rire; mais elle est plus subtile qu'elle n'en a l'air. Car une femme qui a ellemême fait des romans ne peut ignorer à ce point toutes les transformations que subit forcément le réel dès qu'on tâche de le saisir dans une œuvre. Adèle sait pourtant, sans vouloir l'admettre, que la vérité historique compte moins justement que cette ambiance "de poésie" qu'excelle à créer Gabrielle. L'étude que fait par ailleurs Adèle de La Petite Poule d'Eau montre en effet qu'elle comprend les rouages de l'imagination et les sources de l'inspiration; son refus d'en tenir compte est grandiose et grotesque, à la mesure de ses exigences inconscientes. En effet, ce refus obstiné de distinguer le roman du manuel d'histoire ou du guide touristi- 
que masque un enjeu autrement plus important: imposer une esthétique et une éthique de l'écriture complètement opposées à celles de Gabrielle. Adèle, en somme, comprend la fiction; mais elle se défend d'en faire. De tout son cour, elle la refuse, comme on repousse le Mal. Car si la vérité devient le critère essentiel d'évaluation de tout écrit, même de la fiction littéraire, Adèle aura prouvé de façon éclatante et définitive (du moins à ses propres yeux) la supériorité absolue de son ouvre sur celle de Gabrielle. Tout comme l'altruisme d'Adèle a justifié son manque d'audace dans la vie, cette survalorisation de la vérité compense son manque de talent pour l'écriture.

Ainsi, les écrits d'Adèle sont dénués des qualités qui enchantent les lecteurs de Gabrielle? C'est qu'elle les aura volontairement, violemment, rejetées. Gabrielle a toujours été frivole, primesautière, enjôleuse; Adèle, elle, est solide, responsable, vraie. On ne pourra plus alléguer que ses ouvres manquent de charme, de légèreté, de poésie, de fantaisie, car elle-même a réduit ces qualités à néant. Seuls comptent la vérité, l'exactitude, le travail, le sérieux, le "rideau levé sur la réalité": les écrits d'Adèle, en somme.

Ce chapitre des Capucins de Toutes-Aides est précieux dans la mesure où il montre clairement à l'œuvre cet acte de redéfinition de l'écriture qui console Adèle de son insuccès. Écrire, c'est dire la vérité; Gabrielle, qui écrit des faussetés, n'est donc pas une écrivaine véritable. Tout se passe comme si c'était ce besoin de condamner Gabrielle, et tout ce qui se rattache à elle, qui a poussé Adèle à abandonner le roman au profit des ouvrages d'histoire. Ainsi, les enjeux seront clairs: les livres de Gabrielle sont un tissu de fabrications, affirme Adèle; les miens sont vrais du début à la fin ("levons le rideau sur la réalité"). À une vieille méfiance des clercs envers le roman, transmise durant les études auprès des religieuses, à laquelle s'ajoute le souvenir de l'agacement du père devant les fabrications maternelles, Adèle superpose son propre jugement, définitif: la fiction, c'est le mal, c'est le mensonge, c'est... Gabrielle.

\section{Écrire pour les autres ou pour soi ?}

On a souvent relevé les attitudes opposées des deux sœurs envers l'écriture: l'une qui insiste sur la vérité des faits, l'autre qui transforme le réel grâce à l'imagination créatrice ${ }^{13}$. Cette diffé- 
62

rence pourrait bien avoir pour origine l'influence réciproque du père et de la mère: Adèle, fidèle à l'exemple paternel, répugnerait à toute déformation des faits; Gabrielle, à l'instar de la mère, songerait davantage à l'effet produit et aux exigences internes de l'histoire $^{14}$. Mais on pourrait expliquer autrement cette opposition, du moins dans la perspective d'Adèle: là où Adèle a tout à gagner de la révélation des faits et des gestes réels, Gabrielle, pour bien paraître, doit tout embellir, à commencer par sa propre image; elle est donc condamnée à faire de la fiction, bref à mentir. Si la vérité éclatait, Adèle triompherait enfin, croit-elle; malheureusement, les gens préfèrent, de loin, les fictions de Gabrielle.

Gabrielle, affirme encore Adèle, n'a aucune vocation littéraire; elle ne cherche qu'à emberlificoter ses lecteurs pour recevoir en échange, dans ses "mains avides", des "centaines de milliers de dollars" ( $M P$, p. 123). Adèle, en contraste, affirme écrire non pas pour elle mais pour les autres: par exemple, Le pain de chez nous est "un humble et fragile ouvrage qui [rend] honneur et respect à mes parents et à mes grands-parents" ( $M P$, p. 197). L'amour filial et patriotique, plutôt que l'ambition, guide alors sa plume. Combien plus encore Adèle s'efface-t-elle en faisant le récit des Capucins de Toutes-Aides, en chantant leur gloire au lieu de vanter ses propres mérites comme le fait Gabrielle? Sans doute le genre romanesque en est-il venu, avec le temps par la faute, toujours, de Gabrielle —, à lui paraître aussi narcissique que faux. Adèle a donc forgé une vision transitive, toute utilitaire de l'écriture, comme en témoigne aussi le fait que nombre de ses manuscrits inédits ont une destinataire (Gabrielle ou Clémence, par exemple). Adèle parle comme si ses souvenirs d'enfance relevaient de la vérité pure (de la Vérité, écrit-elle) plutôt que de la reconstruction; elle entreprend même de "prouver" que Gabrielle n'a pas eu une enfance malheureuse, comme elle l'affirme pour se justifier et se rendre intéressante, et de lui "faire comprendre ce que ses parents ont fait pour elle ${ }^{15}$ ".

L'équation est donc lapidaire: Adèle = bonne fille = bonne écrivaine; Gabrielle $=$ mauvaise fille $=$ mauvaise écrivaine. La fiction devient une forme de mensonge; Adèle se donne pour mis-

13 Voir par exemple Paul Genuist, op. cit., p. 78.

14 François Ricard, op. cit., p. 46-47.

15 Paul Genuist, op. cit., p. 73. 
sion sacrée de rétablir la vérité familiale et historique. À une esthétique de l'écriture se voit liée une éthique de la vie: Gabrielle s'y trouve deux fois perdante. Adèle triomphe dans sa tête, à défaut de triompher dans la vie.

\section{GABRIELLE}

On ne s'étonnera pas de voir que Gabrielle joue un plus grand rôle dans l'univers imaginaire d'Adèle que l'inverse. En fait, pendant très longtemps, portée par les succès et les honneurs, Gabrielle a plus ou moins oublié sa sœur. Elle en parle peu dans ses ouvres. Un premier portrait, anonyme quoique très flatteur, apparaît dans un reportage du Bulletin des agriculteurs, au moment éphémère où les deux sours se sont rapprochées avant de commencer à s'éloigner pour de bon: la culture et "l'admirable courage" de l'institutrice y sont louées ${ }^{16}$. Plus tard, ce sera sans doute la Georgianna de "Pour empêcher un mariage", cette jeune fille vivante qui court à sa perte, et aussi, de façon moins flatteuse, la vieille maîtresse d'école de La Petite Poule d'Eau:

La pauvre fille s'en allait sans ressentir beaucoup de soulagement après tout. Ce ne serait pas mieux ailleurs. Depuis vingtcinq ans, elle se trimbalait de poste en poste, et celui vers lequel elle allait était toujours un peu plus reculé, un peu plus enfoncé dans la solitude; la nourriture y était de plus en plus lourde, les sentiments de moins en moins délicats, la reconnaissance de plus en plus rare ${ }^{17}$.

Quel contraste avec la jeune, pimpante et adorable Mlle Côté, inspirée de Gabrielle elle-même! Au moins un écrit de Gabrielle, une nouvelle inédite intitulée d'abord "Un air de famille", puis "La maison rose près du bac ${ }^{18}$ ", montre le rôle de repoussoir qu'ont joué ses sœurs à ses yeux, l'une coincée dans un mariage médiocre, l'autre condamnée à la misère et à la solitude; dans la première version, au titre significatif, la protagoniste, Gilberte, craint de se mettre à leur ressembler si elle n'arrive pas à s'arracher à son milieu étouffant et mesquin. Ce texte traduit donc des

16 Gabrielle Roy, "Les gens de chez-nous", Bulletin des agriculteurs, mai 1943, p. 38.

17 Gabrielle Roy, La Petite Poule d'Eau, Montréal, Boréal, 1993, p. 99.

18 Textes inédits de Gabrielle Roy, Bibliothèque nationale du Canada, boîte 71, chemises 2, 3, 4, 17, 18 . 
64

angoisses absentes de l'œuvre publiée et montre que la supériorité souriante qu'affichera Gabrielle au faîte de son succès n'a pas toujours été la sienne. Par ailleurs, le dénouement de cette nouvelle - des retrouvailles entre les trois sœurs devenues vieilles, longtemps après la mort de la mère —, apparaît comme un souhait ou une nostalgie de ce qui n'a jamais existé ${ }^{19}$.

On peut donc croire que Gabrielle se sent supérieure à ses sœurs, puisqu'elle a réussi là où elles ont échoué. Elle représente sans doute pour Adèle, comme Christine pour Éveline dans La route d'Altamont, "quelqu'un d'infiniment mieux que moi que j'aurais pu être ${ }^{20}$ ". Voilà précisément ce qu'Adèle ne supporte pas, ne peut supporter. Une mère, à la rigueur, acceptera de voir sa fille la dépasser, voire la mépriser quelque peu; une sœur, jamais, surtout si elle a caressé les mêmes ambitions. C'est le monde à l'envers: la méchante belle-sœur rafle le prince à Cendrillon dont les mérites restent dans l'ombre, le vilain petit canard ne devient jamais cygne. En effet, jamais Adèle ne sera récompensée de ses fidèles services envers la famille. Elle restera pauvre, obscure, vilaine; Gabrielle est et demeurera riche, célèbre, belle.

Alors que faire pour ne plus passer inaperçue? Comment rétablir la justice? Comment faire pour que Gabrielle prenne enfin sa grande sœur au sérieux? Fine stratège, Adèle attaquera Gabrielle là où elle est vulnérable: dans l'image de grande dame généreuse et noble de cour qu'elle s'est donnée d'elle-même et qu'elle tente d'imposer aux autres. Les lettres de Gabrielle à Bernadette montrent bien combien le dépôt dans une bibliothèque universitaire, par Adèle, d'un manuscrit semblable au Miroir du passé ${ }^{21}$, la plonge dans le désarroi. Elle demande à Bernadette de le détruire si elle en a l'occasion et fait tout pour que l'accès en soit interdit aux chercheurs. Bref, elle remue ciel et terre, panique, parle de "honte" et d'une "menace" qui plane sur

19 Dans La route d'Altamont, où le même type de voyage est effectué par la mère, accompagnée cette fois de la seule Christine, Gabrielle fait comme si ses sours ne comptaient plus pour elle, n'étaient plus, en somme, "du voyage".

20 Gabrielle Roy, La route d'Altamont, Montréal, Boréal, 1993, p. 145.

21 Rédigé vers 1962 et déposé à la bibliothèque de l'Université de Montréal en 1968, "Les deux sources de l'inspiration: l'imagination et le cour" traite essentiellement la même matière que le texte publié en 1979 sous le titre Le miroir du passé. Voir François Ricard, op. cit., p. 438-445. 
toute la famille ${ }^{22}$. Adèle aura réussi : enfin, Gabrielle pense à elle, écrit sur elle, souffre à cause d'elle.

Ce qui intrigue, dans cette histoire en soi mesquine, c'est le nouveau découpage qu'opère alors Gabrielle du champ réalitéfiction. Adèle prétend dévoiler la vérité sur Gabrielle? Il n'y a là que délire d'une vieille fille un peu maniaque, répond la cadette. Le manuscrit d'Adèle est un tissu de faussetés ("elle s'exonère toujours elle-même pour toujours charger les autres", écrit Gabrielle non sans justesse [ $L B$, p. 138]), la vérité ne s'y trouve pas. Incapable de dire la vérité, Adèle n'est pas apte non plus à faire de la fiction, car elle n'aura "jamais le don d'intéresser vraiment les autres, et comment l'aurait-elle, pauvre créature malade, qui vit totalement repliée sur soi?" ( $L B$, p. 138). Autrement dit, là où Adèle dénonçait les fictions mensongères de Gabrielle et renonçait au roman pour mieux célébrer la Vérité, Gabrielle s'arroge à la fois le monopole de la vérité et celui de la fiction; la "pauvre Adèle" n'a plus que la folie ${ }^{23}$. Cette fois-ci, c'est Gabrielle qui remporte la manche, non sans une souffrance intime toutefois, car, lucide à propos des autres à défaut de voir clair en elle-même, Adèle aura visé en partie juste. Ses reproches ne sont pas si fous, et cela, Gabrielle le sait. Le monument qu'est La détresse et l'enchantement est bien, entre autres, une réponse aux accusations d'Adèle, dont Gabrielle ne sent que trop la vérité.

\section{ADÈLE ET GABRIELLE}

\section{À qui appartiennent les histoires?}

L'accusation de vol et de plagiat que formule Adèle contre Gabrielle, après la publication de Rue Deschambault, mérite que nous y revenions, non pas parce que cette accusation est fondée ${ }^{24}$, mais bien parce qu'elle soulève la question, fascinante et

22 Gabrielle Roy, Ma chère petite sœur (Lettres à Bernadette 1943-1970), Montréal, Boréal, 1988, p. 137, 136. Cette ouvre sera désignée dans le corps du texte par l'abréviation suivante: $L B$.

23 L'accusation de folie surgit de part et d'autre, puisque, selon Adèle, le docteur Etsel dit à Anna: "Votre sour Gabrielle est une névropathe qui exagère ses malaises. Elle est le jouet de ses phantasmes!" (MP, p. 143).

24 Au contraire, cette accusation "relève du délire" (François Ricard, op. cit., p. 359). Ricard précise également (p. 356) que les Éditions du Lévrier acceptent le manuscrit d'Adèle "moyennant le versement d'une somme de 
66

complexe, de l'origine des histoires. De quoi Adèle accuse-t-elle Gabrielle au juste? De lui avoir pris, non la manière de raconter qui est fort différente ${ }^{25}$, mais bien la matière de son récit, c'est-àdire les histoires qui l'ont inspirée:

Gabrielle avait puisé dans "Mes souvenirs", dans les lettres d'Anna, la matière de plusieurs de ses nouvelles et de ses contes. Elle racontait, embellis par des trouvailles de mots, des faits survenus avant sa naissance (MP, p. 194).

Gabrielle ignorait tout de ce passé du père, car elle n'était pas née quand il l'évoquait pour nous, les plus âgés, en ces soirs d'hiver (p. 200).

Mais le cœur de la Rue Deschambault, c'était précisément Le Pain de chez nous! Gabrielle Roy décrit un paysage qu'elle ne pouvait avoir vu avec les yeux du corps; car, tel l'agneau de la fable, elle n'était pas née lors de notre emménagement dans ce quartier de la ville [...] La véritable Rue Deschambault, c'est celle que je décris dans Le Pain de chez nous (p. 206, Adèle souligne).

Outre le monopole de la vérité qu'Adèle s'est arrogé depuis longtemps, ainsi que nous l'avons vu, ces passages frappent par l'idée persistante que les récits appartiennent à quelqu'un, sans quoi ils ne pourraient être volés. Mais à qui au juste? Le passé appartientil seulement à celui qui l'a vécu, ou encore à ceux à qui il le raconte, surtout s'ils entreprennent, à sa place, d'en transmettre le récit à la postérité? Peut-on ainsi "donner" sa vie, léguer son histoire, en espérant qu'il en restera quelque chose? Selon Adèle, les récits oraux du père, qu'elle a transcrits dans Le pain de chez nous, lui appartiennent. Mais on voit mal au nom de quoi, car, si Adèle a le droit de puiser dans les souvenirs que conserve son père d'événements qu'elle-même n'a pas vécus, pourquoi Gabrielle ne l'aurait-elle pas? Ce qu'Adèle ne veut pas reconnâ̂tre, c'est qu'elle-même, autant que Gabrielle, s'est approprié l'histoire du père; seulement, elle l'a fait avec infiniment moins de talent que Gabrielle. Adèle invoque donc un hypothétique droit d'aînesse en vertu duquel Gabrielle, née trop tard, se voit privée

750 dollars et à la condition, stipulée au contrat, que "le nom de famille Roy apparaisse comme signature de l'ouvrage" ". Autrement dit, Adèle ne réussit à publier qu'en raison de sa parenté avec Gabrielle et à compte d'auteur.

25 Comme nous l'avons vu plus haut, Adèle considère sa manière de raconter supérieure à celle de Gabrielle en raison de son plus grand respect de la Vérité des faits. 
des "bonnes" histoires de la famille Roy ${ }^{26}$. Seule cette notion d'exclusivité (elle m'a volé mon histoire) protège Adèle contre l'échec. De la même façon, laisser entendre que Gabrielle est plagiaire parce qu'elle reprend les récits familiaux "embellis par des trouvailles de mots" (MP, p. 194), fait bon marché de la qualité littéraire (le fond - les récits - l'emportant haut la main sur la forme - les "trouvailles de mots") et excuse les faiblesses stylistiques d'Adèle ${ }^{27}$.

En effet, à côté de la prose de Gabrielle, celle d'Adèle est laborieuse et sans vie. Non seulement elle ne sait rien dépeindre de façon prenante et dramatique, mais son roman tient de l'hagiographie familiale: elle y pousse à l'extrême, quitte à frôler le ridicule, le culte des aïeux, de la tradition, de la prière. On ne trouvera pas trace, dans l'œuvre de Gabrielle, de ce pesant éloge des valeurs ancestrales, de ce culte de la maison paternelle et des plus infimes reliques familiales. Les deux sœurs se démarquent également l'une de l'autre par leurs portraits respectifs de la mère : Adèle l'accuse de fourberie et de cruauté envers le père, de mollesse envers les autres enfants et (surtout) de complaisance excessive envers Gaétane-Gabrielle; elle relève l'ignorance de cette femme, ses fautes de grammaire et d'orthographe, voire sa vulgarité. Tandis qu'Éveline, dans Rue Deschambault, est une grande dame polie et raffinée, au verbe châtié, un être sans défaut ou presque ${ }^{28}$ : après tout, n'a-t-elle pas eu le bon goût de préférer sa benjamine à tous ses autres enfants? Du reste, cette image idéalisée de la mère en dame du monde correspond trait pour trait à celle que Gabrielle donnera d'elle-même. Inversement, Adèle idéalise davantage le père et s'identifie, de façon assez transparente, à sa droiture, à son bon cour, à sa passion pour la vérité. Comme lui, surtout, elle sera mal comprise et maltraitée par une famille ingrate. Est-ce pour cette raison qu'Adèle dépeint l'autorité masculine comme foncièrement bénigne; est-ce pour

26 On entend peut-être un lointain écho de toute cette histoire de vol et de droit de propriété lorsqu'Éveline raconte aux enfants de son frère Majorique que "dans notre famille, on n'empiétait pas sur les histoires qui étaient censées appartenir à l'une de nous". Voir De quoi t'ennuies-tu, Éveline?, Montréal, Boréal compact, 1988, p. 73.

27 On peut relire les extraits mis en exergue du présent article pour se faire une idée des esthétiques respectives des deux sœurs.

28 Sinon son désir de retenir sa fille auprès d'elle; mais ce n'est pas une faiblesse de caractère. 
cela que la "maison paternelle" évoque chez elle non pas le patriarcat mais l'identification et la légitimité? Est-ce pour se venger d'avoir été moins aimée de la mère que Gabrielle qu'elle durcit le ton lorsqu'elle parle de Mélina et insiste sur sa faiblesse et son absence de clairvoyance?

Autre différence entre les deux univers romanesques: chez Adèle, la vie familiale est faite de tensions, d'accusations et de coups bas, alors que, de façon contradictoire, elle célèbre le culte de la race canadienne-française et la transmission des valeurs de pères en fils. Inversement, dans Rue Deschambault, rien n'évoque l'éloge de la prière en famille et du dur labeur de la glèbe; en revanche, la famille Roy, désunie dans la réalité selon tous les témoins, donne lieu à une fiction familiale souriante, paisible, d'où les conflits entre les enfants ont été pour l'essentiel bannis tandis que la lignée grand-mère-mère-fille est célébrée, voire mythifiée ${ }^{29}$. Si Adèle insiste sur les différends, Gabrielle, elle, les gomme avec soin. Pudeur, mauvaise conscience, peur de se donner le mauvais rôle ou magnanimité réelle? Quoi qu'il en soit, le contraste entre les deux approches ne peut manquer de frapper: celle de Gabrielle, comme toujours, est la plus habile et la plus apte à lui gagner un public admiratif. Car combien plus attirante, au fond, est son attitude de confiance infinie et d'amour fraternel (Luzina Tousignant, par exemple, découvre avec ravissement que "la nature humaine est partout excellente ${ }^{30}$ ") que les charges hargneuses d'Adèle, méfiante et hypercritique. Encore là, la pauvre Adèle fera peu d'adeptes.

\section{Un double roman familial}

Ainsi, les contrastes entre les deux sœurs semblent, de prime abord, plus importants que les convergences. Il n'en reste pas moins que de nombreux traits rapprochent les sœurs ennemies.

L'histoire littéraire a laissé relativement peu de modèles de femmes créatrices en général et de sœurs écrivaines en particu-

29 Dans La saga d'Éveline, roman inachevé et inédit (Bibliothèque nationale du Canada), Gabrielle présente une version plus sombre des relations familiales: les parents ne se reconnaissent pas dans leurs enfants, ni les enfants dans les parents; la plupart des enfants d'Éveline sont clairement destinés au malheur.

30 Gabrielle Roy, La Petite Poule d'Eau, op. cit., p. 30. 
lier. L'exemple qui vient le plus volontiers à l'esprit, celui des sœurs Brontë, pourra toutefois servir, toutes proportions gardées par ailleurs, de point de comparaison. Or malgré la grande intimité qui les a liées tout au long de leur vie (proches en âge, elles ne se sont guère quittées), chacune des søurs Brontë a une esthétique et des sujets propres: on ne pourrait jamais confondre le réalisme visionnaire de Charlotte avec le pragmatisme didactique d'Anne, encore moins avec le lyrisme parfois hallucinatoire d'Emily. Dans le cas de Gabrielle et d'Adèle, en revanche, si les styles sont fort différents, le même fond familial nourrit les deux femmes et crée des parentés évidentes tant dans leur caractère que dans leurs écrits.

Tout d'abord, les deux sœurs - ainsi qu'Anna — se ressemblent par leur tempérant fort et affirmé, leur volonté d'échapper à la tutelle familiale et aux contraintes du milieu pour mener une vie libre et heureuse, et donc, forcément, par leur rejet du modèle maternel ${ }^{31}$. D'où, plus tard, la rancune de celles qui n'ont pas réussi envers celle qui a triomphé au-delà de toute espérance. Que Gabrielle ait payé cher son succès, Adèle n'aura jamais réellement voulu l'entendre ${ }^{32}$; son propre échec relatif, en regard de ses aspirations de jeune fille, ne peut être justifié que par le rappel constant de sa bonté.

Le destin d'Adèle peut donc se voir comme l'envers de celui de Gabrielle, et vice versa. L'une est partie et s'est réalisée; l'autre est restée et a secouru leur mère ${ }^{33}$. Si Gabrielle regrettera toujours l'abandon de la mère et enviera Adèle de l'avoir davantage aidée, Adèle, bien sûr, regrettera l'audace qu'elle n'a pas eue. On se retrouve devant une sorte de symétrie dans la différence, chacune placée devant celle qu'elle aurait pu être et traçant son propre portrait en réaction.

Toutes deux, également, se réclament de la filiation maternelle afin de justifier leur désir d'écrire. On a parfois l'impression de deux petites filles qui jouent à "c'est moi la préférée de maman", chacune s'efforçant, au fond, de montrer (et de se montrer) combien elle a

31 Voir François Ricard, op. cit., p. 58, 60, et Paul Genuist, op. cit.

32 Tout comme chacune des sœurs aînées de "La maison rose près du bac" se plaît à se dépeindre plus malheureuse que l'autre.

33 Encore là, c'est la version d'Adèle, que Gabrielle a en quelque sorte intériorisée; en fait, Adèle est partie beaucoup plus jeune et n'est pas non plus revenue souvent. 
été bonne envers la mère. Adèle l'a soutenue financièrement, nous l'avons vu; mais Gabrielle pourra dire qu'elle aura été une bonne fille aussi, du moins après la mort de la mère, car qui, autant qu'elle, aura chanté sa mère dans ses livres? Elle les lui destine presque tous, elle immortalise la mère afin de racheter quelque peu le tort qu'elle lui a fait ${ }^{34}$. Ce n'est que dans les vibrants hommages de Gabrielle que la mère morte continue à vivre et à rayonner.

Le sentiment dominant, chez Gabrielle, demeure la culpabilité envers sa mère et, plus généralement, envers les siens qu'elle a peu aidés; chez Adèle, on retrouve plutôt un profond sentiment d'injustice: "Je suis une femme de lettres peu chanceuse, poursuivant, en dépit de déceptions, un métier ingrat et peu lucratif, et souvent la cible de mauvais critiques et d'envieux ${ }^{35}$ ". De ces deux sentiments, très différents, procède pourtant un autre trait, déjà mentionné, qui relie les deux sœurs: un profond besoin d'autojustification. L'une doit rendre compte de son départ, l'autre de son insuccès. Adèle parle de son abnégation et de sa grandeur d'âme et ne s'avouerait jamais mesquine, ni autoritaire, ni jalouse $^{36}$; par ailleurs, si ses œuvres n'ont pas connu la gloire, c'est par la faute des journalistes mesquins, des éditeurs cupides et, bien sûr, de sa sour. Quant à Gabrielle, on sait combien l'écriture lui a permis de reconstruire son image d'elle-même, si malmenée par la vie en général et par Adèle en particulier; mais aucune réussite littéraire et mondaine ne sera assez grande pour racheter à ses yeux son abandon de la mère. Le projet d'écriture de l'une comme de l'autre sœur s'appuie fortement sur ce besoin de (se) prouver qu'on a été, qu'on est la meilleure. L'image d'un moi idéalisé se trouvera donc au cour de leurs univers romanesques, d'où un narcissisme profond que Gabrielle transcende grâce à la beauté de son écriture, mais que rien ne compense chez Adèle.

Au-delà des visées autobiographiques qu'elles partagent également ${ }^{37}$, ayant toutes deux signé autant des romans autobiogra-

34 Voir "Gabrielle Roy: écriture et réparation", Lori Saint-Martin, Le nom de la mère. Mères, filles et écriture dans la fiction québécoise au féminin, Québec, Nota bene, 1999.

35 "Voyages en Europe", manuscrit inédit, p. 18, cité par Paul Genuist, op. cit., p. 70.

36 Pour les relations orageuses d'Adèle avec ses frères et soeurs - elle veut bien leur prodiguer des dons d'argent à condition qu'ils acceptent aussi avec reconnaissance ses conseils -, voir Paul Genuist, op. cit.

37 Paul Genuist, op. cit., p. 2. 
phiques qu'une autobiographie véritable, un autre désir réunit Adèle et Gabrielle: le vaste projet de mettre en scène et en texte l'histoire familiale. En effet, toutes les deux seront presque obsédées par cette histoire et y consacreront de longues années de leur vie. Ici, il faut bien le dire, c'est Adèle, pour une fois, qui a réussi : du moins, elle a achevé et publié deux romans et des ouvrages historiques là où Gabrielle, au bout d'intenses efforts, ne réussira jamais à terminer son grand livre sur la famille, La saga d'Éveline. On peut imaginer qu'Adèle aurait trouvé là une vengeance ultime, car elle a réussi précisément là où Gabrielle a échoué. Gabrielle semble s'être trompée sur ses aptitudes en voulant écrire cette saga familiale; le tempérament d'historien, de chroniqueur des temps passés, elle le possède peu, malgré son intérêt durable pour le sujet. Elle a consacré à cette œuvre, en pure perte, de longues années de sa vie. Il se peut qu'un tel projet, proche de la vérité historique et extérieure plutôt que personnelle et intime, convienne mal à sa propre manière de procéder par invention, amalgame, alchimie, alors que le tempérament pragmatique et tatillon d'Adèle, ainsi que sa manie pour les recherches en archives, la prédisposent à une telle entreprise. Pour une fois, en tout cas, c'est Adèle qui l'emporte.

\section{Comment devient-on écrivain ?}

Peu après la publication de Bonheur d'occasion, Anna écrit à Adèle: "Grand Dieu! Que nous ne puissions, toi et moi, écrire aussi bien, mieux qu'elle. C'est là que seraient les vengeances. Mais hélas! il aurait fallu comme elle se servir des gens comme marche-pieds - il aurait fallu qu'on eût une roche à la place du coeur ${ }^{38}$ ". Tout le drame des soeurs Roy est là, au fond, entre la vengeance et l'égoïsme. À lire sur la vie des grands créateurs, on se demande parfois si le sacrifice d'autrui - le sacrifice, au fond, de tout ce qui n'est pas l'écriture - n'est pas au contraire une condition quasi nécessaire pour bâtir une œuvre.

Ainsi, on ne peut se donner aux autres et en même temps à son ouvre, affirme Gabrielle. Adèle insiste sur l'égoïsme personnel de Gabrielle; celle-ci affirme tenir moins à elle-même qu'à sa mission d'écriture. "Ah, si tu savais ce qu'un seul conte, une seule

38 Lettre d'Anna à Adèle, 1948, cité par Paul Genuist, op. cit., p. 131. 
minute d'inspiration coûte souvent d'oubli de soi et de lourds sacrifices ${ }^{39}$ ", écrit-elle à son mari. Gabrielle a tout sacrifié pour l'écriture, y compris sa propre tranquillité d'esprit, a tout abandonné pour la servir, y compris sa bonne image d'elle-même et le bonheur de sa mère. Adèle n'a pas eu cette volonté obsessionnelle, ce but unique et tout-puissant qui l'a absorbée entièrement ; de ce point de vue, elle n'a pas mérité le succès. Tout se passe comme si Gabrielle Roy avait accepté de tout donner à l'écriture - sa personne, son bonheur, sa vie en somme - par regret de ne pas avoir consenti ce sacrifice à sa mère pendant qu'il était encore temps. Et pourtant, après coup, le sacrifice, le don même entier ne sera jamais suffisant.

Égoïsme, mérite, bonté, sacrifice: l'enjeu entre les deux sours est moral plutôt qu'esthétique. On revient encore et toujours à la question d'être la bonne fille de la mère. Un dernier trait, au fond, rapproche les deux sœurs: une même passion malheureuse l'une pour l'autre, jusqu'à la fin de la vie. Gabrielle redoute Adèle, qui s'acharne à la tourmenter; Adèle, toujours rongée par le succès de Gabrielle, désire à la fois son pardon et la reconnaissance du fait qu'elle-même avait raison. C'est une relation passionnelle, surtout peut-être du côté d'Adèle, mais elle obsède également Gabrielle et l'atteint à la fois dans les fibres de son être (elle se sent réellement coupable, comme Adèle affirme qu'elle le devrait) et dans le personnage public qu'elle s'est créé au bout de longs et patients efforts. Rien n'est plus désolant, en tout cas, que l'image de deux vieilles femmes brouillées depuis des décennies - elles se verront pour la dernière fois en $1954^{40}$ - et néanmoins liées par une lutte terrible, chacune tentant d'imposer au monde sa vision de soi-même et de l'autre, éloignées l'une de l'autre par cela même qui les rapproche: leurs regrets respectifs et opposés.

39 Lettre à Marcel Carbotte, Genève, 31 janvier 1948, citée par François Ricard, op. cit., p. 313.

40 François Ricard, op. cit., p. 360. 